\title{
On Bianchi Type III String Cloud Universe Containing Strange Quark Matter
}

\author{
Kangujam Priyokumar Singh1, Koijam Manihar Singh ${ }^{2}$ \\ ${ }^{1}$ Department of Mathematical Sciences, Bodoland University, Kokrajhar, India \\ ${ }^{2}$ Department of Mathematics, National Institute of Technology, Imphal, India \\ Email: pk mathematics@yahoo.co.in, maniharskoijam@gmail.com
}

Received 25 July 2014; revised 18 August 2014; accepted 15 September 2014

Copyright (C) 2014 by authors and Scientific Research Publishing Inc.

This work is licensed under the Creative Commons Attribution International License (CC BY).

http://creativecommons.org/licenses/by/4.0/

(c) (i) Open Access

\begin{abstract}
Considering Bianchi type III space-time we present the model Universe containing strange quark matter which is expanding, anisotropic, with a sign of dark energy that help in accelerated expansion of this Universe. It is also seen that the model Universe contains both particles and strings but ultimately will have fluid containing particles only. This model which we consider here is acceptable in view of the present observations of the Universe. Some physical and geometrical properties are also discussed.
\end{abstract}

\section{Keywords}

Dark Energy, Evolution, String Clouds, Early Universe, Dark Matter

\section{Introduction}

Several aspects of strange quark matter have been investigated by many researchers. This work is a continuation of [1]. The origin of our Universe is one of the greatest cosmological mysteries even today. The exact physical situation at early stage of the formation of our Universe is still a subject of study. The concept of string theory was developed to describe events of the early stage of the evolution of the Universe. The general relativistic treatment of strings was initiated by [2] and [3]. The gravitational effects of cosmic strings have been extensively discussed by [4] [5] in general relativity. Relativistic string models in the context of Bianchi space time have been obtained by [6]-[9].

Here we suppose that strange quark matters are attached to the string cloud. It is plausible to attach strange quark matter to the string cloud, because, one of such transitions during the phase transitions of the universe could be Quark Glucon Plasma (QGP) hadron gas (called quark-hadron phase transition) when cosmic temperature was $T \approx 200 \mathrm{Mev}$. [10]-[12] have formed two ways for creation of strange quark matter. One is the 
quark-hadron phase transition in the early Universe and another in the conversion of neutron stars into strange ones at ultrahigh density. In strong interaction theories it is supposed that breaking of physical vacuum takes place inside hadrons to form quark bag model.

As a result vacuum energy densities inside and outside a hadron become essentially different, and the vacuum pressure on the bag wall equilibrates the pressure of quarks, thus stabilizing the system. [13] [14] examined that if the hypothesis of the quark matter is true, then some of neutron stars could actually be strange stars built entirely of strange matter. [15] studied models of strange quark matter attached to the string cloud in the spherical symmetric space-time admitting conformal motion discussing strange star properties. Also [16]-[18] studied 5-D Kaluza-Klein cosmological models with quark matter attached to the string cloud and domain walls.

The strange quark matter is modelled with an equation of state based on the phenomenological bag model of quark matter, in which quark confinement is described by an energy term proportional to the volume. In this model, quarks are through as degenerate Fermi gas, which exists only in a region of space endowed with a vacuum energy density $B_{c}$ (called as the bag constant). In the framework of this model, the quark matter is composed of massless $u$ and d quarks, massive s quarks and electrons. [19] discussed charged strange quark matter in the spherically symmetric space-time admitting conformal motion. [20] has discussed string cloud and domain walls with quark matter in n-dimensional Kaluza-Klien cosmological model in general relativity and strange quark matter attached to string cloud in Bianchi type-III space time in general relativity. [21] has confirmed their work with quark matter which is attached to the topological defects in general relativity. [22] has obtained cosmological model with strange quark matter attached to cosmic strings for axially symmetric space-time in general relativity. Recently, [23] has discussed geometry of quark and strange quark matter in higher dimensional general relativity. [24] has discussed about higher dimensional cosmological model with quark and strange quark matter. [25] discussed Bianchi Type II, VIII and IX cosmological models with strange quark matter attached to string cloud in B-D and general theory of gravitation.

Here we study the model universe containing strange quark matter which is expanding, anisotropic, with a sign of dark energy that help in accelerated expansion of this universe. It is also seen that the model universe which we discuss contains both particles and strings, but ultimately will have fluid containing particles only. The model which we consider here is acceptable in view of the present observations of the Universe. The physical interpretations of the solutions obtained are also discussed.

\section{Field Equations and Their Solutions}

We consider the line element of Bianchi type III universe

$$
\mathrm{d} s^{2}=\mathrm{d} t^{2}-A^{2} \mathrm{~d} x^{2}-B^{2} \mathrm{e}^{-2 a x} \mathrm{~d} y^{2}-C^{2} \mathrm{~d} z^{2}
$$

where the metric potentials $A, B$, and $C$ are the functions of time " $t$ " alone and " $a$ " is non-zero constant. For string cloud the energy momentum is given by

$$
T_{i j}=\rho u_{i} u_{j}-\rho_{s} x_{i} x_{j}
$$

where $\rho$ is the rest energy density for the cloud of strings with particle attached to them, $\rho_{p}$ is the particle energy density and $\rho_{s}$ is the string tension density such that

$$
\rho=\rho_{p}+\rho_{s}
$$

We assume quarks are massless and non-interacting as in Bag model.

So, quark pressure is

$$
p_{q}=\frac{\rho_{q}}{3}
$$

where $\rho_{q}$ is quark energy density.

Total energy density is

$$
\rho=\rho_{q}+B_{c}
$$

and total pressure is

$$
p=p_{q}-B_{c}
$$


For this universe containing strange quark matter we will consider quarks instead of particles in the string cloud, so we consider the energy density of quark matter instead of the energy density of the particles in the string cloud.

From Equations (3) and (5), we obtain

$$
\rho=\rho_{q}+\rho_{s}+B_{c}
$$

Here field equations are

$$
\begin{gathered}
\frac{\ddot{B}}{B}+\frac{\ddot{C}}{C}+\frac{\dot{B} \dot{C}}{B C}=0 \\
\frac{\ddot{A}}{A}+\frac{\ddot{C}}{C}+\frac{\dot{A} \dot{C}}{A C}=0 \\
\frac{\ddot{A}}{A}+\frac{\ddot{B}}{B}+\frac{\dot{A} \dot{B}}{A B}-\frac{a^{2}}{A^{2}}=\rho_{s} \\
\frac{\dot{A} \dot{B}}{A B}+\frac{\dot{A} \dot{C}}{A C}+\frac{\dot{B} \dot{C}}{B C}-\frac{a^{2}}{A^{2}}=\rho \\
\frac{\dot{A}}{A}-\frac{\dot{B}}{B}=0
\end{gathered}
$$

Here we get as solutions

$$
\begin{gathered}
A=\left[b_{1} \cos (\sqrt{3} b t)+b_{2} \sin (\sqrt{3} b t)\right] \mathrm{e}^{b t} \\
B=c_{0}\left[b_{1} \cos (\sqrt{3} b t)+b_{2} \sin (\sqrt{3} b t)\right] \mathrm{e}^{b t} \\
C=b_{0} \mathrm{e}^{-2 b t}
\end{gathered}
$$

where $b, b_{0}, b_{1}, b_{2}, c_{0}$ are arbitrary constants.

Therefore here,

$$
\begin{aligned}
\rho_{s}= & 2 \frac{\ddot{A}}{A}+\left(\frac{\dot{A}}{A}\right)^{2}-\frac{a^{2}}{A^{2}} \\
& =\frac{4 b^{2}\left(\sqrt{3} b_{2}-b_{1}\right) \cos (\sqrt{3} b t)-4 b^{2}\left(\sqrt{3} b_{1}+b_{2}\right) \sin (\sqrt{3} b t)}{b_{1} \cos (\sqrt{3} b t)+b_{2} \sin (\sqrt{3} b t)} \\
& +\frac{\left[\left(b b_{1}+\sqrt{3} b b_{2}\right) \cos (\sqrt{3} b t)+\left(b b_{2}-\sqrt{3} b b_{1}\right) \sin (\sqrt{3} b t)\right]^{2}-a^{2}}{\left[b_{1} \cos (\sqrt{3} b t)+b_{2} \sin (\sqrt{3} b t)\right]^{2}} . \\
\rho & =\left(\frac{\dot{A}}{A}\right)^{2}+2 \frac{\dot{A} \dot{C}}{A C}-\frac{a^{2}}{A^{2}} \\
= & \frac{\left[\left(b b_{1}+\sqrt{3} b b_{2}\right) \cos (\sqrt{3} b t)+\left(b b_{2}-\sqrt{3} b b_{1}\right) \sin (\sqrt{3} b t)\right]^{2}-a^{2}}{\left[b_{1} \cos (\sqrt{3} b t)+b_{2} \sin (\sqrt{3} b t)\right]^{2}} \\
& -2 b \frac{\left[\left(b b_{1}+\sqrt{3} b b_{2}\right) \cos (\sqrt{3} b t)+\left(b b_{2}-\sqrt{3} b b_{1}\right) \sin (\sqrt{3} b t)\right]^{2}}{b_{1} \cos (\sqrt{3} b t)+b_{2} \sin (\sqrt{3} b t)} .
\end{aligned}
$$




$$
\begin{aligned}
& \rho_{p}=\frac{4 b^{2}\left(\sqrt{3} b_{1}+b_{2}\right) \sin (\sqrt{3} b t)-4 b^{2}\left(b_{1}-\sqrt{3} b_{2}\right) \cos (\sqrt{3} b t)}{b_{1} \cos (\sqrt{3} b t)+b_{2} \sin (\sqrt{3} b t)} \\
& -\frac{2 b\left[\left(b b_{1}+\sqrt{3} b b_{2}\right) \cos (\sqrt{3} b t)+\left(b b_{2}-\sqrt{3} b b_{1}\right) \sin (\sqrt{3} b t)\right]}{b_{1} \cos (\sqrt{3} b t)+b_{2} \sin (\sqrt{3} b t)} \\
& =\frac{2 b^{2}\left(2 b_{2}+2 \sqrt{3} b_{1}+\sqrt{3} b_{1}-b_{2}\right) \sin (\sqrt{3} b t)-2 b^{2}\left(-2 \sqrt{3} b_{2}+2 b_{1}+b_{2}+\sqrt{3} b_{2}\right) \cos (\sqrt{3} b t)}{b_{1} \cos (\sqrt{3} b t)+b_{2} \sin (\sqrt{3} b t)} \text {. } \\
& \theta=\frac{\dot{A}}{A}+\frac{\dot{B}}{B}+\frac{\dot{C}}{C}=\frac{2 \sqrt{3} b b_{2} \cos (\sqrt{3} b t)-2 \sqrt{3} b b_{1} \sin (\sqrt{3} b t)}{b_{1} \cos (\sqrt{3} b t)+b_{2} \sin (\sqrt{3} b t)}
\end{aligned}
$$

And

$$
\begin{gathered}
\sigma^{2}=\frac{1}{3}\left[3 \frac{\left\{\left(b b_{1}+\sqrt{3} b b_{2}\right) \cos (\sqrt{3} b t)+\left(b b_{2}-\sqrt{3} b b_{1}\right) \sin (\sqrt{3} b t)\right\}^{2}}{\left\{b_{1} \cos (\sqrt{3} b t)+b_{2} \sin (\sqrt{3} b t)\right\}^{2}}+4 b^{2}\right. \\
\left.+4 b \frac{\left(b b_{1}+\sqrt{3} b b_{2}\right) \cos (\sqrt{3} b t)+\left(b b_{2}-\sqrt{3} b b_{1}\right) \sin (\sqrt{3} b t)}{b_{1} \cos (\sqrt{3} b t)+b_{2} \sin (\sqrt{3} b t)}\right] .
\end{gathered}
$$

From above here,

$$
\begin{gathered}
\rho_{p}=\frac{\left(2 \sqrt{3} b^{2} b_{2}-6 b^{2} b_{1}\right) \cos (\sqrt{3} b t)+\left(6 \sqrt{3} b^{2} b_{1}+2 b^{2} b_{2}\right) \sin (\sqrt{3} b t)}{b_{1} \cos (\sqrt{3} b t)+b_{2} \sin (\sqrt{3} b t)} \\
v=b_{0} c_{0}\left[b_{1} \cos (\sqrt{3} b t)+b_{2} \sin (\sqrt{3} b t)\right]^{2} \\
q=-\left[1-\frac{3\left(b_{1}^{2}+b_{2}^{2}\right)}{2 b^{2}}\left\{\frac{b_{1} \cos (\sqrt{3} b t)+b_{2} \sin (\sqrt{3} b t)}{b_{2} \cos (\sqrt{3} b t)-b_{1} \sin (\sqrt{3} b t)}\right\}\right] \\
H=\frac{2 b}{\sqrt{3}} \times\left\{\frac{b_{2} \cos (\sqrt{3} b t)-b_{1} \sin (\sqrt{3} b t)}{b_{1} \cos (\sqrt{3} b t)+b_{2} \sin (\sqrt{3} b t)}\right\}
\end{gathered}
$$

Quark energy density

$$
\rho_{q}=\rho_{p}-B_{c}=\frac{2 b^{2}\left(b_{2}+3 \sqrt{3} b_{1}\right) \sin (\sqrt{3} b t)+2 b^{2}\left(\sqrt{3} b_{2}-3 b_{1}\right) \cos (\sqrt{3} b t)}{b_{1} \cos (\sqrt{3} b t)+b_{2} \sin (\sqrt{3} b t)}-B_{c}
$$

where $B_{c}$ is the bag constant.

$$
p_{q}=\frac{2 b^{2}\left(b_{2}+3 \sqrt{3} b_{1}\right) \sin (\sqrt{3} b t)+2 b^{2}\left(\sqrt{3} b_{2}-3 b_{1}\right) \cos (\sqrt{3} b t)}{3\left[b_{1} \cos (\sqrt{3} b t)+b_{2} \sin (\sqrt{3} b t)\right]}-\frac{B_{c}}{3}
$$

Here

$$
H_{x} \equiv \frac{\dot{A}}{A}, \quad H_{y} \equiv \frac{\dot{B}}{B}, \quad \text { and } \quad H_{z} \equiv \frac{\dot{C}}{C}
$$




$$
\begin{gathered}
H_{x}=b+\frac{\sqrt{3} b b_{2} \cos (\sqrt{3} b t)-\sqrt{3} b b_{1} \sin (\sqrt{3} b t)}{b_{1} \cos (\sqrt{3} b t)+b_{2} \sin (\sqrt{3} b t)} \\
H_{y}=b+\frac{\sqrt{3} b b_{2} \cos (\sqrt{3} b t)-\sqrt{3} b b_{1} \sin (\sqrt{3} b t)}{b_{1} \cos (\sqrt{3} b t)+b_{2} \sin (\sqrt{3} b t)} \\
H_{z}=-2 b
\end{gathered}
$$

Anisotropy parameter of the expansion

$$
\begin{gathered}
\Delta=\frac{2}{9} \times \frac{1}{H^{2}}\left(H_{x}-H_{y}\right)^{2} \\
=\frac{2}{9} \times \frac{3}{4 b^{2}} \times \frac{\left[\sqrt{3} b\left\{b_{2} \cos (\sqrt{3} b t)-b_{1} \sin (\sqrt{3} b t)\right\}+3 b\left\{b_{1} \cos (\sqrt{3} b t)+b_{2} \sin (\sqrt{3} b t)\right\}\right]^{2}}{\left[b_{2} \cos (\sqrt{3} b t)-b_{1} \sin (\sqrt{3} b t)\right]^{2}} \\
p=-\left[\frac{2 b}{3}\left\{b_{1} \cos (\sqrt{3} b t)+b_{2} \sin (\sqrt{3} b t)\right\}\left\{\left(b b_{1}+\sqrt{3} b b_{2}\right) \cos (\sqrt{3} b t)+\left(b b_{2}-\sqrt{3} b b_{1}\right) \sin (\sqrt{3} b t)\right\}+\left(\frac{a^{2}}{3}\right)\right. \\
\left.-\frac{1}{3}\left\{\left(b b_{1}+\sqrt{3} b b_{2}\right) \cos (\sqrt{3} b t)+\left(b b_{2}-\sqrt{3} b b_{1}\right) \sin (\sqrt{3} b t)\right\}^{2}\right] \div\left[b_{1} \cos (\sqrt{3} b t)+b_{2} \sin (\sqrt{3} b t)\right]^{2}-\frac{4 B_{c}}{3} .
\end{gathered}
$$

\section{Physical Interpretations of the Solutions}

In this model, from the values of deceleration parameter $q$ and expansion factor $\theta$ we see that our model Universe is expanding at an accelerated rate. And since, here $\frac{\sigma}{\theta} \neq 0$, our model is found to be an anisotropic one. The volume of this model Universe is found to increase rapidly with time, thus giving testimony to the accelerated expansion of this model. This model Universe is also found to be a shearing one.

The pressure of our model Universe is also found to be negative which is a characteristic of this model Universe to be a dark energy model and which helps in the accelerated expansion of the Universe. A negative pressure here stimulates a repulsive gravity and inflates the Universe by overwhelming the usual gravitational effects of matter, thereby playing an important role in getting an accelerated expansion of the Universe.

In this model Universe the quark energy density as well as the quark pressure is found to be increasing functions of time. Here the string density is found to be a decreasing function of time whereas the particle density is found to be an increasing function of time showing that this model Universe will contain both particles and strings but ultimately will be a fluid containing particle only. The pressure and energy density of the fluid are seen to be negligible at late time, and this is acceptable in view of the present observations of the Universe.

It can be seen that even though the fluid in this model Universe produces an anisotropic parameter, its anisotropy does not promote anisotropy in the expansion. Rather, since anisotropic of fluid isotropises at late times of the Universe in accelerating models, our Universe will approach to isotropy as it is an accelerating model.

\section{References}

[1] Adhav, K.S., Nimkar, A.S., Raut, V.B. and Thakare, R.S. (2009) Strange Quark Matter Attached to String Cloud in Bianchi Type-III Space Time. Astrophysics and Space Science, 319, 81-84. http://dx.doi.org/10.1007/s10509-008-9941-1

[2] Stachel, J. (1980) Thickening the String I. The String Perfect Dust. Physics Review D, 21, 2171. http://dx.doi.org/10.1103/PhysRevD.21.2171

[3] Letelier, P.S. (1983) String Cosmologies. Physics Review D, 28, 2414. http://dx.doi.org/10.1103/PhysRevD.28.2414

[4] Vilenkin, A. (1981) Gravitational Field of Vacuum Domain Walls and Strings. Physics Review D, $23,852$. http://dx.doi.org/10.1103/PhysRevD.23.852 
[5] Gott, J.R. (1985) Gravitational Lensing Effects of Vacuum Strings—Exact Solutions. Astrophysical Journal, Part 1, 288, 422-427. http://dx.doi.org/10.1086/162808

[6] Krori, K.D., Chaudhury, T., Mahanta, C.R. and Mazumdar, A. (1990) Some Exact Solutions in String Cosmology. General Relativity and Gravitation, 22, 123-130. http://dx.doi.org/10.1007/BF00756203

[7] Banerjee, S. and Bhui, B. (1990) Homogeneous Cosmological Model in Higher Dimension. Monthly Notices of the Royal Astronomical Society, London, 247, 57.

[8] Tikekar, R. and Patel, L.K. (1994) A Class of Cylindrically Symmetrical Models in String Cosmology. General Relativity and Gravitation, 26, 647-653. http://dx.doi.org/10.1007/BF02116954

[9] Bhattacharjee, R. and Baruah, K.K. (2001) String Cosmologies with a Scalar Field. Pure and Applied Mathematics, 32, 47.

[10] Itoh, N. (1970) Hydrostatic Equilibrium of Hypothetical Quark Stars. Progress of Theoretical Physics, 44, $291-292$. http://dx.doi.org/10.1143/PTP.44.291

[11] Bodmer, A.R. (1971) Collapsed Nuclei. Physics Review D, 4, 1601-1606. http://dx.doi.org/10.1103/PhysRevD.4.1601

[12] Witten, E. (1984) Cosmic Separation of Phases. Physics Review D, 30, 272. http://dx.doi.org/10.1103/PhysRevD.30.272

[13] Alcock, C., Farhi, E. and Olinto, A. (1986) Strange Stars. Astrophysical Journal, 310, 261-272. http://dx.doi.org/10.1086/164679

[14] Haensel, P., Zdunik, J.L. and Schaeffer, R. (1986) Strange Quark Stars. Astronomy and Astrophysics, 160, $121-128$.

[15] Cheng, K.S., Dai, Z.G. and Lu, T. (1998) Strange Stars and Related Astrophysical Phenomena. International Journal of Modern Physics D, 7, 139. http://dx.doi.org/10.1142/S0218271898000139

[16] Yavuz, I., Yilmaz, I. and Baysal, H. (2005) Strange Quark Matter Attached to the String Cloud in the Spherical Symmetric Space-Time Admitting Conformal Motion. International Journal of Modern Physics D, 14, 1365. http://dx.doi.org/10.1142/S0218271805007061

[17] Yilmaz, I. (2005) Domain Wall Solutions in the Nonstatic and Stationary Godel Universes with a Cosmological Constant. Physics Review D, 71, 103503. http://dx.doi.org/10.1103/PhysRevD.71.103503

[18] Yilmaz, I. (2006) String Cloud and Domain Walls with Quark Matter in 5-D KaluzaKlein Cosmological Model. General Relativity and Gravitation, 38, 1397-1406. http://dx.doi.org/10.1007/s10714-006-0322-1

[19] Mak, M.K. and Harko, T. (2004) Quark Stars Admitting a One-Parameter Group of Conformal Motions. International Journal of Modern Physics D, 13, 149. http://dx.doi.org/10.1142/S0218271804004451

[20] Adhav, K.S., Nimkar, A.S. and Dawande, M.V. (2008) String Cloud and Domain Walls with Quark Matter in n-Dimensional Kaluza-Klein Cosmological Model. International Journal of Theoretical Physics, 47, 2002-2010. http://dx.doi.org/10.1007/s10773-007-9644-3

[21] Khadekar, G.S. and Wanjari, R. (2009) Domain Wall with Strange Quark Matter in Kaluza-Klein Type Cosmological Model. International Journal of Theoretical Physics, 48, 2550-2557. http://dx.doi.org/10.1007/s10773-009-0040-z

[22] Katore, S.D. and Shaikh, A.Y. (2012) Cosmological Model with Strange Quark Matter Attached to Cosmic String for Axially Symmetric Space-Time. International Journal of Theoretical Physics, 51, 1881-1888. http://dx.doi.org/10.1007/s10773-011-1064-8

[23] Khadekar, G.S. and Rupali, W. (2012) Geometry of Quark and Strange Quark Matter in Higher Dimensional General Relativity. International Journal of Theoretical Physics, 51, 1408-1415. http://dx.doi.org/10.1007/s10773-011-1016-3

[24] Khadekar, G.S. and Rajani, S. (2012) Higher Dimensional Cosmological Model with Quark and Strange Quark Matter. International Journal of Theoretical Physics, 51, 1442-1447. http://dx.doi.org/10.1007/s10773-011-1020-7

[25] Rao, V.U.M. and Sireesha, K.V.S. (2013) Axially Symmetric Space-Time with Strange Quark Matter Attached to String Cloud in Brans-Dicke Theory of Gravitation. International Journal of Theoretical Physics, 52, 1052-1060. http://dx.doi.org/10.1007/s10773-012-1420-3 
Scientific Research Publishing (SCIRP) is one of the largest Open Access journal publishers. It is currently publishing more than 200 open access, online, peer-reviewed journals covering a wide range of academic disciplines. SCIRP serves the worldwide academic communities and contributes to the progress and application of science with its publication.

Other selected journals from SCIRP are listed as below. Submit your manuscript to us via either submit@scirp.org or Online Submission Portal.
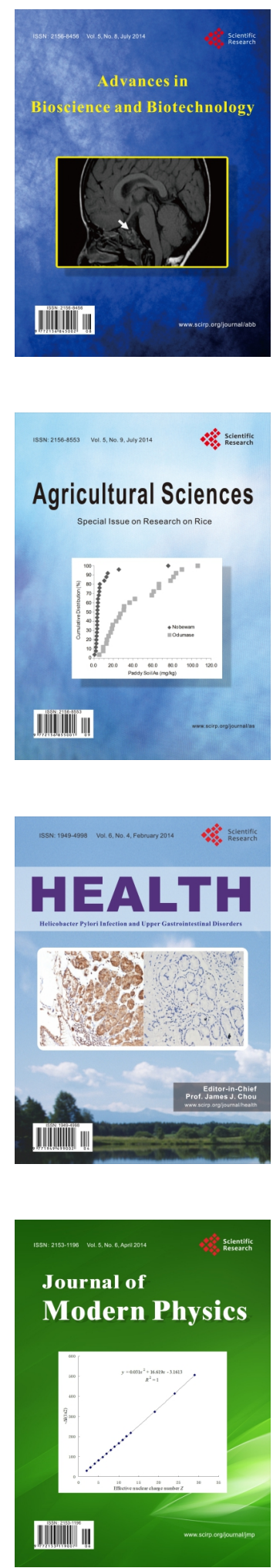
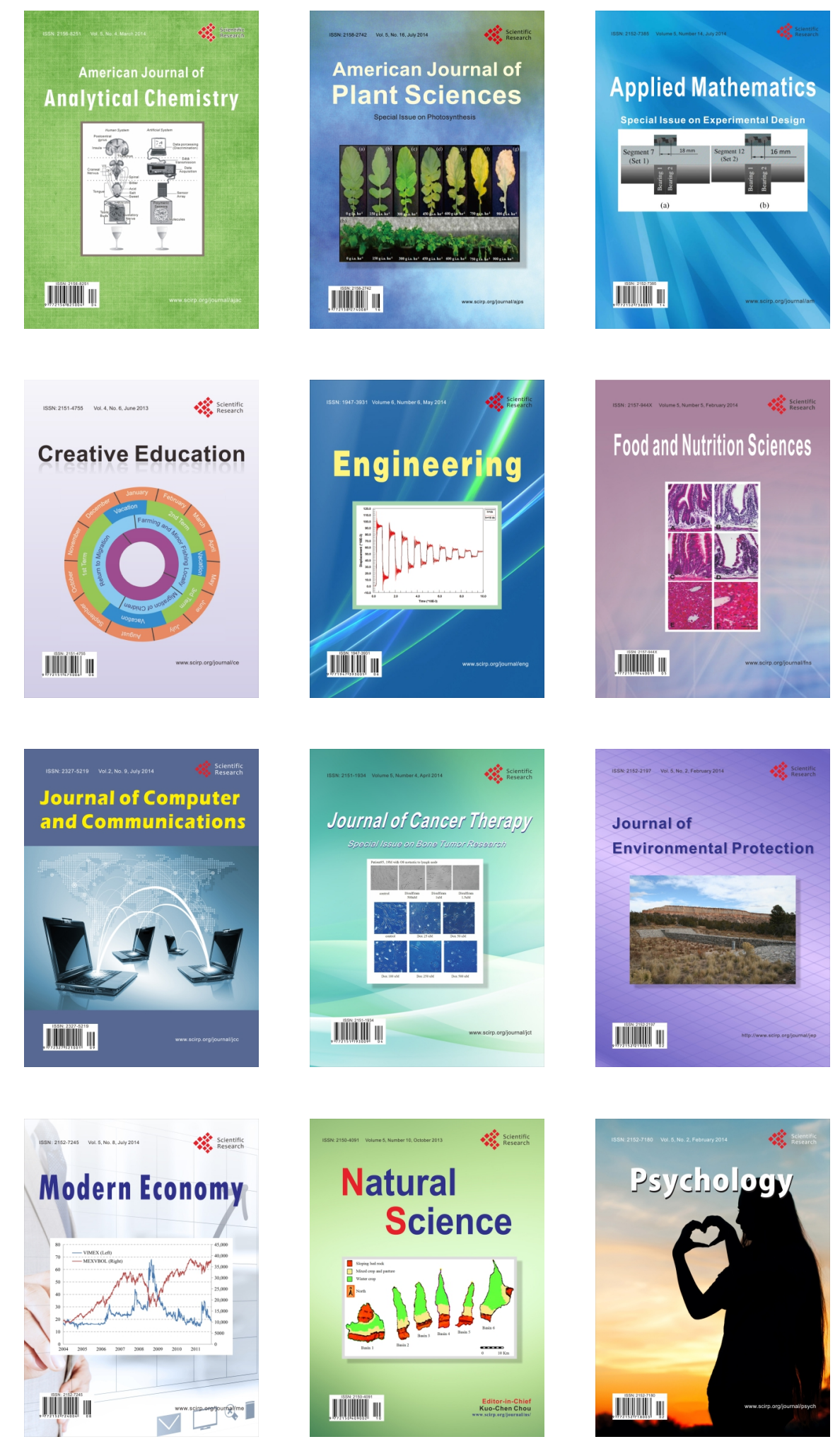